\title{
Taste Quality Representation in the Human Brain
}

\author{
기ason A. Avery, Alexander G. Liu, John E. Ingeholm, Cameron D. Riddell, Stephen J. Gotts, and Alex Martin \\ Laboratory of Brain and Cognition, National Institute of Mental Health, Bethesda, Maryland 20892
}

In the mammalian brain, the insula is the primary cortical substrate involved in the perception of taste. Recent imaging studies in rodents have identified a "gustotopic" organization in the insula, whereby distinct insula regions are selectively responsive to one of the five basic tastes. However, numerous studies in monkeys have reported that gustatory cortical neurons are broadly-tuned to multiple tastes, and tastes are not represented in discrete spatial locations. Neuroimaging studies in humans have thus far been unable to discern between these two models, though this may be because of the relatively low spatial resolution used in taste studies to date. In the present study, we examined the spatial representation of taste within the human brain using ultra-high resolution functional magnetic resonance imaging (MRI) at high magnetic field strength (7-tesla). During scanning, male and female participants tasted sweet, salty, sour, and tasteless liquids, delivered via a custom-built MRI-compatible tastant-delivery system. Our univariate analyses revealed that all tastes (vs tasteless) activated primary taste cortex within the bilateral dorsal mid-insula, but no brain region exhibited a consistent preference for any individual taste. However, our multivariate searchlight analyses were able to reliably decode the identity of distinct tastes within those mid-insula regions, as well as brain regions involved in affect and reward, such as the striatum, orbitofrontal cortex, and amygdala. These results suggest that taste quality is not represented topographically, but by a distributed population code, both within primary taste cortex as well as regions involved in processing the hedonic and aversive properties of taste.

Key words: 7T; fMRI; MVPA; taste

\section{Significance Statement}

The insula is the primary cortical substrate involved in taste perception, yet some question remains as to whether this region represents distinct tastes topographically or via a population code. Using high field (7-tesla), high-resolution functional magnetic resonance imaging in humans, we examined the representation of different tastes delivered during scanning. All tastes activated primary taste cortex within the bilateral mid-insula, but no brain region exhibited any consistent taste preference. However, multivariate analyses reliably decoded taste quality within the bilateral mid-insula as well as the striatum, orbitofrontal cortex, and bilateral amygdala. This suggests that taste quality is represented by a spatial population code within regions involved in sensory and appetitive properties of taste.

\section{Introduction}

The sensation of taste begins on the tongue with sensory receptor cells tuned to one of the five basic tastes: sweet, salty, sour, bitter, and umami (Roper and Chaudhari, 2017). These taste receptor cells signal to afferent sensory neurons, whose fibers travel along cranial nerves VII, IX, and X to reach their first synapse at the nucleus of the solitary tract in the medulla (Beckstead et al.,

Received July 19, 2019; revised Oct. 15, 2019; accepted Dec. 3, 2019.

Author contributions: J.A.A., C.D.R., S.J.G., and A.M. designed research; J.A.A., A.G.L., J.E.I., and C.D.R. performed research; J.A.A., A.G.L., and C.D.R. analyzed data; J.A.A., S.J.G., and A.M. wrote the paper.

This work was supported by the Intramural Research Program of the National Institute of Mental Health, National Institutes of Health, and it was conducted under NIH Clinical Study Protocol 10-M-0027 (ZIA MH002920). Clinicaltrials.gov ID: NCT01031407. We thank Sean Marrett, Martin Hebart, the NIMH Section on Instrumentation, and the $\mathrm{NIH}$ Clinical Center pharmacy for their assistance with various aspects of the design and execution of this study.

The authors declare no competing financial interests.

Correspondence should be addressed to Jason A. Avery at jason.avery@nih.gov.

https://doi.org/10.1523/JNEUROSCI.1751-19.2019

Copyright $\odot 2020$ the authors
1980). In primates, these second-order taste afferents are relayed to the thalamic gustatory nucleus (known as either the basal ventral medial nucleus, or the parvocellular portion of the ventroposteromedial nucleus; Pritchard et al., 1986) before reaching their cortical target in the mid-dorsal region of the insular cortex (Small, 2010). The nature of taste coding in the periphery is the subject of some controversy, with some studies supporting a labeled-line model, wherein receptor cells and peripheral neurons are tuned to specific taste modalities (for review, see Chandrashekar et al., 2006), and others supporting an across-fiber model, wherein tastes are represented by a complex combinatorial code across specifically and broadly-tuned peripheral neurons (Tomchik et al., 2007)

At the cortical level, there are two basic models that can account for the representation of taste quality within the insular cortex. Within a topographic model, a specific taste, such as sweet, is represented at a discreet spatial location within gustatory cortex, analogous to somatotopy in the somatosensory system, or 
retinotopy in the visual system. Alternately, within a population coding model, specific tastes are represented by dispersed ensembles of cortical neurons without any clear spatial organization, much like olfactory representations in the piriform cortex (Stettler and Axel, 2009). Currently, although there is some support for the topographical model in rodents (Chen et al., 2011), human neuroimaging studies have failed to provide evidence for this possibility. Many of these previous studies, however, have relied on small sample sizes and relatively low resolution imaging (Schoenfeld et al., 2004; Prinster et al., 2017). Higher resolution neuroimaging methods may be needed to identify topographically distinct insula regions selective for particular tastes. Relatedly, another possibility suggested by a previous human neuroimaging study (Schoenfeld et al., 2004) is that, although distinct tastes may be represented topographically, this representation may not be uniform from one subject to another. If so, then taste topography may not be observable at the group level but may be revealed in individuals scanned on different days.

Unlike retinotopy and somatotopy, where sensory signals are represented according to their well defined location in space, the sensation of taste has no such spatial layout, as previous theories of a taste map on the tongue have been disproven (Chandrashekar et al., 2006). Nor is there any clear evidence for topographic organization within the subcortical taste nuclei in the brainstem or thalamus (Simon et al., 2006). Indeed, many neurophysiology studies in rodents and primates have provided evidence for some form of a population coding model. These studies report that the neurons in gustatory cortex are responsive to a complex variety of orosensory, viscerosensory, and motor signals, with relatively few neurons responsive solely to taste (Scott and Plata-Salamán, 1999; Scott and Giza, 2000). Indeed, in primates, most taste-responsive neurons appear to be broadly tuned to multiple tastes, and there appears to be no observable topographic organization for specific tastes (Scott et al., 1991). If, as these primate studies suggest (Scott and Plata-Salamán, 1999; Kaskan et al., 2019), tastes are represented by ensembles of gustatory cortical neurons without any clear topography, tastespecific neural responses might be discernable within gustatory cortex using multivariate pattern analysis (MVPA) techniques (Kriegeskorte et al., 2006), as previously demonstrated for olfaction in piriform cortex (Howard et al., 2009). In fact, some evidence for this possibility in the human brain has recently been reported (Chikazoe et al., 2019).

To distinguish between these competing models, we examined taste-evoked hemodynamic responses within the human brain using ultra-high resolution functional magnetic resonance imaging (MRI) at high magnetic field strength (7-tesla). During scanning, participants tasted sweet, salty, sour, and tasteless (control) liquids, delivered via a custom-built MRI-compatible tastant-delivery system. To identify brain regions exhibiting shared and distinct responses for each taste, we used both standard univariate analyses as well as multivariate analysis techniques to examine distributed activation patterns across multiple brain voxels.

\section{Materials and Methods}

Participants. Eighteen subjects (11 female) between the ages 22 and 48 (average: 27 years). Ethics approval for this study was granted by the NIH Combined Neuroscience Institutional Review Board under protocol 10M-0027. The institutional review board of the National Institutes of Health approved all procedures, and written informed consent was obtained for all subjects. Participants were excluded from taking part in the study if they had any history of neurological injury, known genetic or medical disorders that may impact the results of neuroimaging, prenatal drug exposure, severely premature birth or birth trauma, current usage of psychotropic medications, or any exclusion criteria for MRI.

Experimental design. All scanning was performed at the NIH Clinical Center in Bethesda, MD. Participant sessions began with a taste assessment performed within the 7T scanner itself, but before the beginning of the scan session. This was done to ensure that ratings were obtained as close in time as possible to the scan itself, without any effects of differing environmental context between the testing and scanning session. The taste assessment was followed by a high-resolution anatomical reference scan and a functional MRI session, during which they performed our Taste Perception task. Five participants returned for a second session where they once again completed the Taste Perception task. The time between these sessions varied from 6 to $98 \mathrm{~d}$ (average $34 \mathrm{~d}$ ).

Taste stimuli. Participants received four tastant solutions during scanning and the pre-scan taste assessment: Sweet ( $0.6 \mathrm{M}$ sucrose), Sour (0.01 $\mathrm{M}$ citric acid), Salty $(0.20 \mathrm{M} \mathrm{NaCl})$, and Neutral $(2.5 \mathrm{~mm} \mathrm{NaHCO} 3+25$ $\mathrm{mm} \mathrm{KCl})$. To reduce within-subject variability, all participants received tastants at the same molar concentration. The specific concentrations used for this study were derived from median concentrations used in previous neuroimaging studies of taste perception, compiled in previous neuroimaging meta-analyses (Veldhuizen et al., 2011; Yeung et al., 2017). All tastants were prepared using sterile laboratory techniques and USP-grade ingredients by the NIH Clinical Center Pharmacy.

Gustometer description. A custom-built pneumatically-driven MRIcompatible system delivered tastants during fMRI scanning (Simmons et al., 2013b; Avery et al., 2015, 2017, 2018; Fig. 1). Tastant solutions were kept at room temperature in pressurized syringes and fluid delivery was controlled by pneumatically-driven pinch-valves that released the solutions into polyurethane tubing that ran to a plastic gustatory manifold attached to the head coil. The tip of the polyethylene mouthpiece was small enough to be comfortably positioned between the subject's teeth. This insured that the tastants were always delivered similarly into the mouth. The pinch valves that released the fluids into the manifold were open and closed by pneumatic valves located in the scan room, which were connected to a stimulus delivery computer, which controlled the precise timing and quantity of tastants dispensed to the subject during the scan. Visual stimuli for behavioral and fMRI tasks were projected onto a screen located inside the scanner bore and viewed through a mirror system mounted on the head-coil. Both visual stimulus presentation and tastant delivery were controlled and synchronized via a custombuilt program developed in the PsychoPy2 environment.

Taste assessment. During blocks of the taste assessment task, the word "Taste" appeared on the screen for $2.5 \mathrm{~s}$, and subjects received $0.5 \mathrm{ml}$ of either a sweet, sour, salty, or neutral tastant (Fig. $1 b$ ). Next, the word "Swallow" appeared on the screen for $2.5 \mathrm{~s}$, prompting subjects to swallow the tastant. Following this, subjects were asked to indicate, using a hand-held response box, (1) the identity of the tastant they received, by selecting it from one of four possible options (sweet, sour, salty, or neutral) presented on the screen; (2) the pleasantness of the taste on a 0 (not pleasant at all) to 10 (extremely pleasant) scale; and (3) the intensity of the taste, on a 0 (not intense at all) to 10 (extremely intense) scale. Subjects provided ratings via a handheld fiber-optic response box (Current Designs). Rating periods were self-paced, average of $\sim 3 \mathrm{~s}$ for rating trials and $\sim 2 \mathrm{~s}$ for identification trials. Following these rating periods, the word "wash" appeared on the screen and subjects received $1.2 \mathrm{ml}$ of the neutral tastant, to rinse out the preceding taste. This was followed by another $(2.5 \mathrm{~s})$ prompt to swallow. A $5 \mathrm{~s}$ fixation period separated successive blocks of the taste assessment task. Tastants were presented five times each (20 blocks total), in random order. Altogether, this session lasted between 5 and $10 \mathrm{~min}$.

Taste perception task. During the Taste Perception fMRI task (Fig. 1c), the word "taste" appeared on the screen for $2.5 \mathrm{~s}$, and subjects received $0.5 \mathrm{ml}$ of either a sweet, sour, salty, or neutral tastant. Next, the word "swallow" appeared on the screen for $2.5 \mathrm{~s}$, prompting subjects to swallow. These taste and swallow periods occurred four times in a row, with the identical tastant delivered each time. Following these four periods, the word "wash" appeared on the screen and subjects received $1.2 \mathrm{ml}$ of the neutral tastant, to rinse out the preceding tastes. This was followed by 

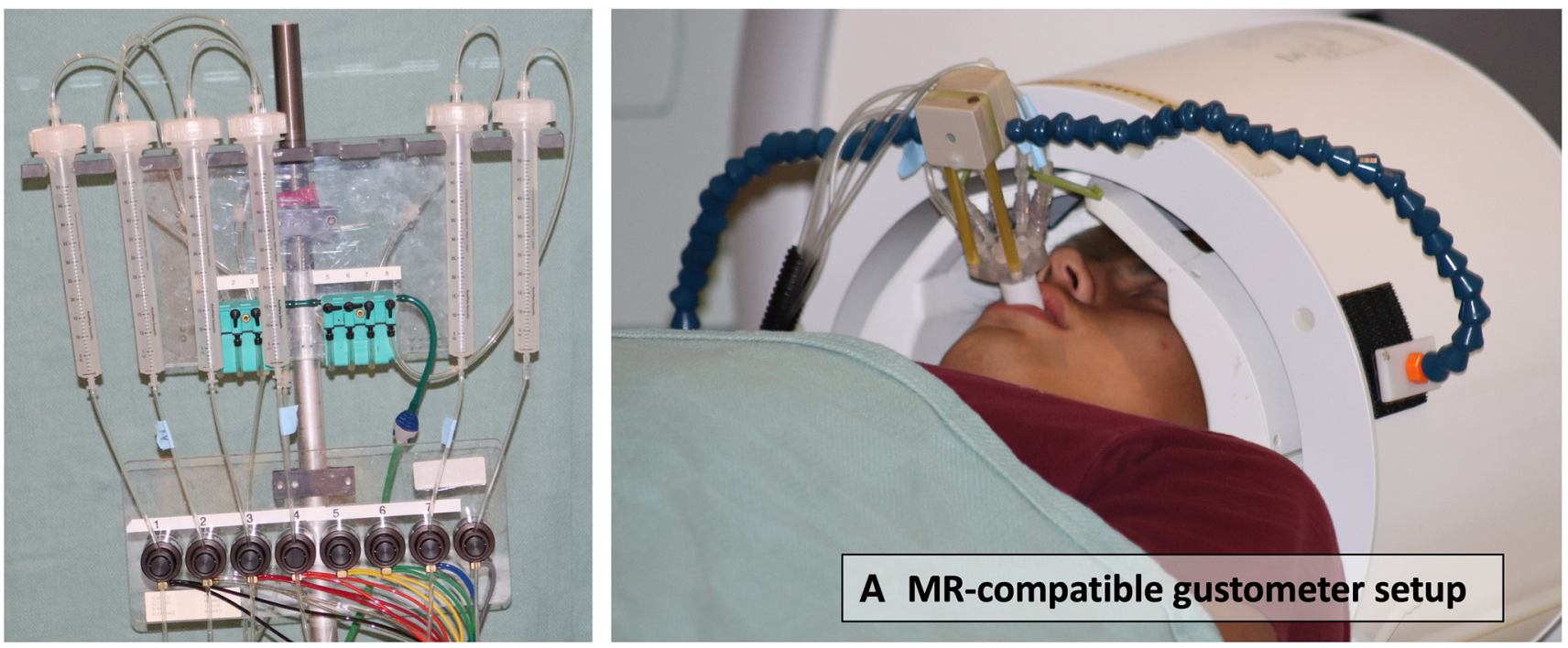

B Behavioral ratings - Pre-scan \begin{tabular}{|c|}
\hline Tastel \\
Swallow \\
\hline
\end{tabular}

$5 s$

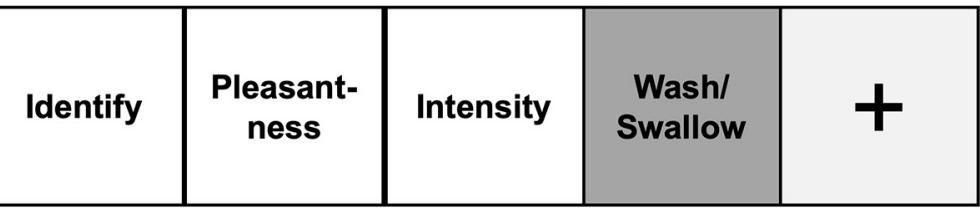

5s

5s

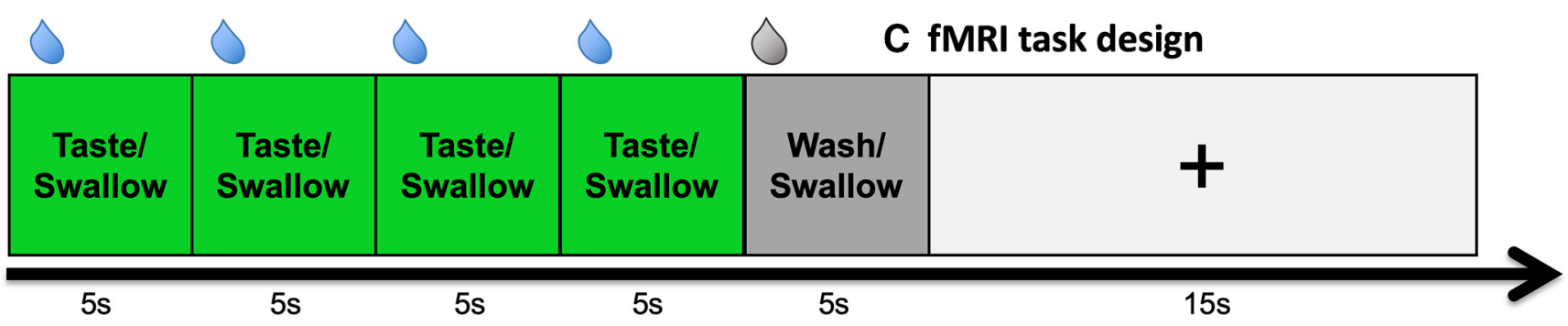

Figure 1. Tastant delivery system and task design. $\boldsymbol{A}$, During scanning, participants tasted sweet, salty, sour, and tasteless liquids, delivered via a custom-built MRI-compatible tastant-delivery system. $\boldsymbol{B}$, While in the scanner, but before fMRI scanning, subjects rated the identity, pleasantness, and intensity of each taste solution. See behavioral results in Figure 1-1, available at https://doi.org/10.1523/JNEUROSCI.1751-19.2019.f1-1.C, During the fMRI task, received $0.5 \mathrm{ml}$ of sweet, sour, salty, and neutral tastant, in a block design (4 identical taste events/block), followed by a wash period. $\mathrm{fMRI}$, functional Magnetic Resonance Imaging.

another $(2.5 \mathrm{~s})$ prompt to swallow. In total, these taste delivery blocks lasted $25 \mathrm{~s}$. Participants were not instructed to perform any ratings during the scanning session. Nor were they asked the identity, pleasantness, or intensity of the tastants. Previous research has identified that the insula is particularly sensitive to attentional orientation (Veldhuizen et al., 2007; Simmons et al., 2013a; Avery et al., 2015, 2017), and taste activity patterns vary significantly within the insula, according to task context (Bender et al., 2009). Following taste delivery blocks, a fixation cross "+" appeared on the screen for $15 \mathrm{~s}$. Two sweet, salty, sour, and neutral taste delivery blocks ( 16 total) were presented in random order throughout each run of this task. Each run lasted $325 \mathrm{~s}$ ( 5 min 25 s). Participants completed eight runs of the Taste Perception task during one scan session.

Imaging methods. fMRI data were collected at the NIMH fMRI core facility at the NIH Clinical Center using a Siemens 7T-830/AS MAGNETOM scanner and a 32-channel head coil. Each volume consisted of $681.2 \mathrm{~mm}$ axial slices [echo time $(\mathrm{TE})=23 \mathrm{~ms}$, repetition time $(\mathrm{TR})=2500 \mathrm{~ms}$, flip angle $=55^{\circ}$, voxel size $=1.2 \times 1.2 \times 1.2 \mathrm{~mm}^{3}$ ]. A Multi-Band factor of 2 was used to acquire data from multiple slices simultaneously. A GRAPPA factor of 2 was used for in-plane slice acceleration along with a $6 / 8$ partial Fourier $k$-space sampling. Each slice was oriented in the axial plane, with an anterior-to-posterior phase encoding direction. The scan window did not achieve full brain coverage though, and data were not acquired from areas at the top of the frontal and parietal lobes, as well as the very bottom of the cerebellum. See Figure 2, $a$ and $c$, for boundaries of echoplanar imaging (EPI) scan window at subject and group level. Before task scans, a 1 min EPI scan was acquired with the opposite phase encoding direction (posterior-to-anterior), which was used for correction of spatial distortion artifacts during preprocessing (see Image preprocessing). An ultra-high resolution MP2RAGE sequence was used to provide an anatomical reference for the $\mathrm{fMRI}$ analysis $\left(\mathrm{TE}=3.02 \mathrm{~ms}, \mathrm{TR}=6000 \mathrm{~ms}\right.$, flip angle $=5^{\circ}$, voxel size $=$ $0.70 \times 0.70 \times 0.70 \mathrm{~mm}$ ).

Image preprocessing. All fMRI preprocessing was performed in AFNI (http://afni.nimh.nih.gov/afni). The FreeSurfer software package (http:// surfer.nmr.mgh.harvard.edu/) was additionally used for skull-stripping the anatomical scans. A de-spiking interpolation algorithm (AFNI's $3 \mathrm{dDespike)}$ was used to remove transient signal spikes from the EPI data, and a slice timing correction was then applied to the volumes of each EPI scan. The EPI scan acquired in the opposite $(\mathrm{P}-\mathrm{A})$ phase encoding direction was used to calculate a nonlinear transformation matrix, which was used to correct for spatial distortion artifacts. All EPI volumes were then 

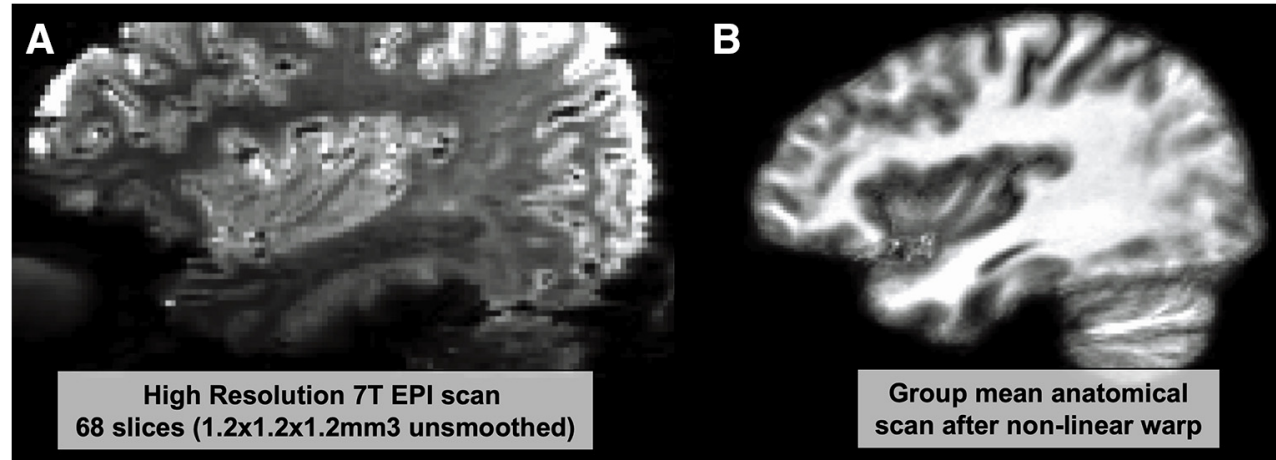

68 slices $(1.2 \times 1.2 \times 1.2 \mathrm{~mm} 3$ unsmoothed) scan after non-linear warp

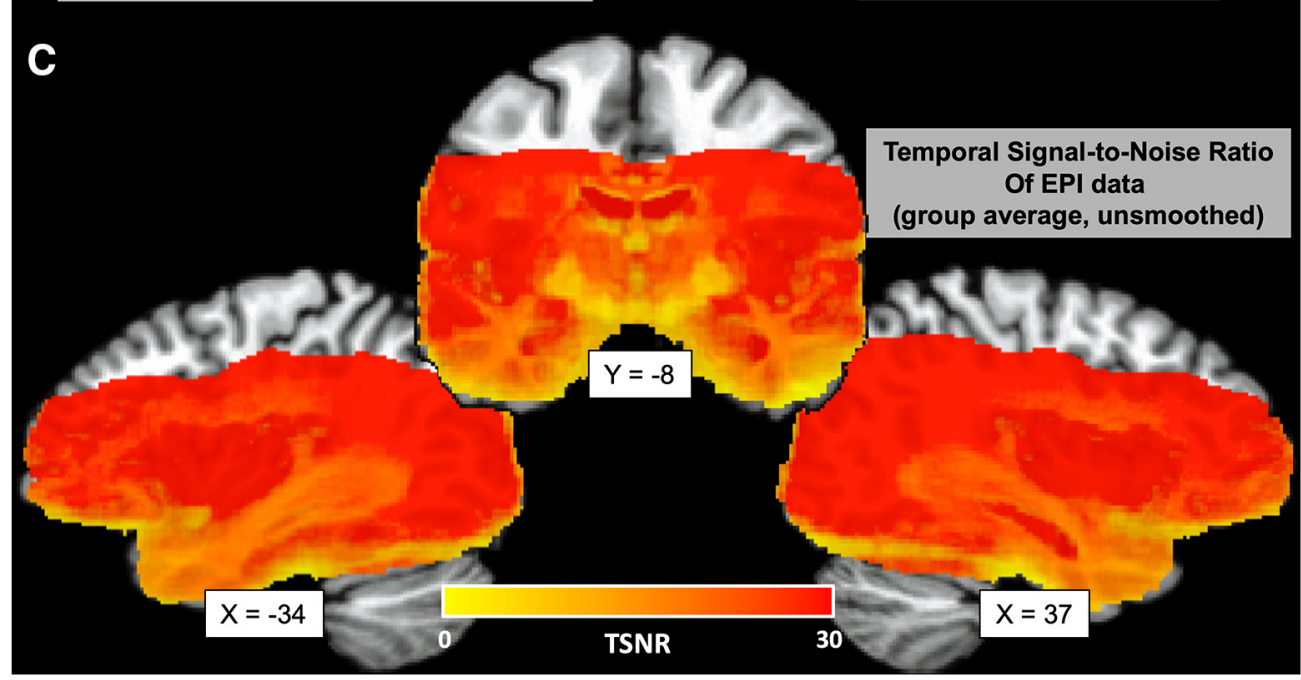

Figure 2. Imaging parameters. Functional MRI data were acquired at ultra-high voxel resolution $(1.2 \times 1.2 \times 1.2 \mathrm{~mm})$ at high magnetic field strength (7-tesla). $A$, EPI scans were acquired in 68 axial slices, in a scan window that ranged from the top of the cingulate gyrus (superiorly) to the tip of the temporal pole (inferiorly). $\boldsymbol{B}$, Anatomical images were transformed to Talairach atlas space using a high-fidelity nonlinear warp. C, Average TSNR of un-blurred EPI images within a mask constructed from the intersection of EPI scan windows for all subjects, after nonlinear transformation to Talairach space. MRI, Magnetic Resonance Imaging; EPI, echoplanar imaging; TSNR, temporal signal-to-noise ratio.

registered to the very first EPI volume using a six-parameter ( 3 translations, 3 rotations) motion correction algorithm, and the motion estimates were saved for use as regressors in the subsequent statistical analyses. Volume registration and spatial distortion correction were implemented in the same nonlinear transformation step, to minimize the number of interpolation steps performed on EPI data. For univariate analyses, smoothing with a $2.4 \mathrm{~mm}$ (i.e., 2-voxel width) full-width at half-maximum (FWHM) Gaussian kernel was used to enhance image signal-to-noise ratio. Importantly, the core univariate results (see Figs. 3, 4) were not qualitatively different when examined with no spatial smoothing applied. No spatial smoothing was used for multivariate analyses. Finally, the signal intensity for each EPI volume was normalized to reflect percentage signal change from each voxel's mean intensity across the time course.

Anatomical scans were first coregistered to the EPI scans and were then spatially normalized to Talairach space via a nonlinear transformation implemented in AFNI's 3dQwarp. These nonlinear transformation matrices were then used to register the subject-level statistical maps to Talairach atlas space. No voxel-size resampling was performed during this registration process, and all data were left at the original resolution $(1.2 \times$ $1.2 \times 1.2 \mathrm{~mm}^{3}$ ).

In addition to volume registration, motion-estimation procedures were also implemented to guard against potential artifactual confounds induced by uncontrolled subject motion. Briefly, the Euclideannormalized derivative of the subject's motion parameters was calculated for each TR, and a list of time points was created in which that value was $>0.3$ ( $\sim 0.3 \mathrm{~mm}$ motion). The TRs within this list were then censored during the subject-level regression analysis. Additionally, any subject with an average Euclidean-normalized derivative of $>0.3$ during the task was excluded from the group-level analysis. Three subjects were thus excluded from the original group of 21 subjects, because of excessive head motion, leaving 18 subjects.

Subject-level regression analysis. The EPI data collected during the Taste Perception task were analyzed at the subject level using multiple linear regression models in AFNI's 3dDeconvolve. The regression model included one regressor for each tastant block (sweet, salty, sour, and neutral) and one regressor for wash/swallow events. These regressors were constructed by convolution of a gamma-variate hemodynamic response function (HRF) with a boxcar function having a $20 \mathrm{~s}$ width ( 5 for wash events) beginning at the onset of each trial period. The regression model also included regressors of non-interest to account for each run's mean, linear, quadratic, and cubic signal trends, as well as the six normalized motion parameters ( 3 translations, 3 rotations) computed during the volume registration preprocessing.

In a supplemental analysis, to more aggressively correct for any swallowing-related motion, we included 24 regressors in the subject-level regression model to account for participant head motion estimated during the volume registration preprocessing (including: the original 6 rigid body motion parameters, the squares of those parameters, the temporal derivatives of those parameters, and the squares of those temporal derivaties). To account for any motion-related artifacts induced by swallowing, we included a gamma-variate HRF for the onset of each swallowing period, as well as a parametrically-modulated HRF whose amplitude varied with the magnitude of head motion (estimated as the Euclidean-normalized derivative of the subject's rigid-body motion parameters) during each individual swallowing event.

Group-level analyses. To evaluate between the competing models of taste representation, we performed both univariate and multivariate analyses on data from the Taste Perception task. For the univariate analyses, we sought to identify brain areas exhibiting both shared and distinct 
Table 1. Pre-scan tastant ratings

\begin{tabular}{|c|c|c|c|c|c|c|c|c|}
\hline \multirow[b]{2}{*}{ Taste } & \multirow{2}{*}{$\begin{array}{l}\text { Pleasantness } \\
\text { Mean (SD) }\end{array}$} & \multicolumn{3}{|c|}{ Taste contrast* } & \multirow{2}{*}{$\begin{array}{l}\text { Intensity } \\
\text { Mean (SD) }\end{array}$} & \multicolumn{3}{|c|}{ Taste contrast* } \\
\hline & & Vs neutral & Vs salty & Vs sour & & Vs neutral & Vs salty & Vs sour \\
\hline Sweet & $6.21(1.41)$ & 0.003 & $<0.001$ & $<0.001$ & $5.46(0.24)$ & $<0.001$ & 0.72 & 0.43 \\
\hline Sour & $3.71(1.53)$ & 0.22 & 0.99 & & $5.03(0.18)$ & 0.001 & 0.47 & \\
\hline Salty & $3.80(1.62)$ & 0.22 & & & $5.55(0.33)$ & $<0.001$ & & \\
\hline Neutral & $4.68(0.67)$ & & & & $2.49(1.74)$ & & & \\
\hline
\end{tabular}

*Bonferroni corrected paired $t$ test.

activation for each taste. All group-level contrast maps created (sweet vs sour, sweet vs salty, salty vs sour, and each taste vs neutral) were separately corrected for multiple comparisons (see Permutation testing for multiple-comparison correction).

First, to identify brain areas exhibiting shared activation for each taste, we used the statistical contrast, all taste (sweet, salty, and sour) versus neutral. For this analysis, we used the subject-level $\beta$ coefficient maps to perform group-level random-effects analyses, using the AFNI program $3 \mathrm{dttest}++$. This contrast was corrected for multiple comparisons using a cluster-size FWE correction using nonparametric permutation tests, described below (see Permutation testing section). Second, to identify brain voxels exhibiting specific taste preferences, we performed a series of conjunctions of our taste contrast maps. For each of our tastes (sweet, salty, and sour), we identified brain voxels where activity for that taste was greater than both other tastes, as well as the neutral tastant. For example, to be considered as sweet-selective the voxels would need to exhibit a significantly greater response to sweet versus sour, versus salty, and versus neutral. The individual contrast maps which were used for these conjunction analyses were analyzed and separately corrected for multiple comparisons, using threshold-free cluster enhancement, implemented in the FSL function "randomise" (Smith and Nichols, 2009).

We performed these conjunction analyses at the level of individual subjects as well, separately correcting the statistical contrast maps that went into each conjunction using a voxelwise FDR correction. In a less stringent approach to identifying differences between tastes at the group level, we also used the AFNI program 3dANOVA2 to identify a main effect of taste from among the three tastants (i.e., sweet, salty, and sour).

Multivariate pattern analyses. Our next analysis approach was to identify whether, within insula regions broadly responsive to multiple tastes, taste-specific neural responses might be discernable using MVPA techniques. The analysis, based on a linear support-vector-machine approach, was trained and tested on subject-level regression coefficient maps obtained from the Taste Perception task, using leave-one-run-out cross-validation. To generate the regression coefficient maps used as input for these analyses, a separate subject-level regression model was applied to the EPI data, which modeled each of the eight task runs separately, so that all task conditions would have eight $\beta$-coefficient maps for the purposes of model training and testing. These analyses were conducted using The Decoding Toolbox (Hebart et al., 2014). The midinsula regions-of-interest (ROIs) used for these analyses were identified as significantly responsive to all tastes versus the tasteless solution, as described.

At the whole-brain level, we ran an MVPA searchlight analysis (Kriegeskorte et al., 2006), which allowed us to identify the average classification accuracy within a multivoxel searchlight, defined as a sphere with a four-voxel radius centered on each voxel in the brain (251 voxels/ $433 \mathrm{~mm}^{3}$ total). These searchlight analyses were again conducted using The Decoding Toolbox (Hebart et al., 2014), and results were tested in AFNI. For every subject, we performed a separate searchlight analysis which evaluated all three pairwise taste contrasts (sweet vs sour, sweet vs salty, salty vs sour). The output of this searchlight analysis was a voxelwise map of average classification accuracy minus chance (33\% for the multiclass comparisons). To evaluate the classification results at the group level, we warped the resulting classification maps to Talairach atlas space, and applied a small amount (2.4 mm FWHM) of spatial smoothing to normalize the distribution of scores across the dataset. We then performed group-level random-effects analyses using the AFNI program $3 \mathrm{dttest}++$ and applied a nonparametric permutation test to correct for multiple comparisons.
Assessment of covariance with pleasantness ratings. In a series of supplemental analyses, we examined the relationship between fMRI activation to tastants and participants' self-reported pleasantness ratings of those tastants in two separate ways. First, we included participants' average pleasantness ratings for each of the tastant solutions (including the neutral solution) in a separate subject-level regression model using parametric modulation regression in AFNI, which varies the height of the HRF by the height of the associated behavioral covariate. In this case, the predicted response to tastant delivery varied as a function of subjects' pleasantness ratings. Importantly, this was distinct from the model described (in Subject-level regression analysis), in which the pleasantness regressor was not included because of collinearity with the individual tastant regressors. In total, this regression model included a single regressor for the onset of all tastant blocks, a parametric regressor for the pleasantness of those tastant blocks, as well as regressors of non-interest for motion, polynomial trend, and wash trials, as described in Subject-level regression analysis. To estimate the amount of within-session variability accounted for by participants' pleasantness ratings, we then performed a group-level random-effects analyses on the parametrically-modulated $\beta$-coefficient maps, using the AFNI program 3 dttest ++ .

Second, we performed a linear mixed-effects (LME) analysis at the group level, using the AFNI program 3dLME (Chen et al., 2013). This analysis was used to estimate the amount of variability accounted for by pleasantness ratings, across subjects. For this analysis, we provided the program with the subject-level $\beta$-coefficient maps for each tastant (including neutral), as well as subjects' pleasantness ratings for each of those tastants.

Permutation testing for multiple-comparison correction. Multiple comparison correction was performed using AFNI's 3dClustSim (AFNI v19.0.25, March 15, 2019), within a whole-volume temporal signal-tonoise ratio (TSNR) mask. This mask was constructed from the intersection of the EPI scan windows for all subjects (after transformation to Talairach space) with a brain mask in atlas space (Fig. $2 c$ ). The mask was then subjected to a TSNR threshold, such that all remaining voxels within the mask had an average un-smoothed TSNR of 10 or greater. For onesample $t$ tests, this program will randomly flip the sign of individual datasets within a sample 10,000 times. This process generates an empirical distribution of cluster-size at the desired voxelwise statistical threshold (in this case, $p<0.001$ ). The clusters which survive correction are those that are larger than $95 \%$ of the clusters within this empirical cluster-size distribution. Permutation tests using cluster size have been demonstrated to accurately control false-positive rates (for discussion, see Eklund et al., 2016).

\section{Results}

\section{Behavioral results}

We identified a main effect of taste intensity $(F=11.1, p<$ 0.001). Our post hoc $t$ tests revealed that the sweet, salty, and sour tastants were each rated as more intense than the neutral tastant ( $p<0.001$, Bonferroni-corrected for multiple comparisons), though they did not differ in intensity among themselves $(p>$ 0.43; Fig. 1-1, available at https://doi.org/10.1523/JNEUROSCI.1751-19.2019.f1-1; Table 1). We also identified a main effect of taste pleasantness $(F=14.4, p<0.001)$, with the sweet tastant rated as the most pleasant [mean $(\mathrm{SD})=6.2$ (1.41); all comparisons: $p<0.001$, followed by the neutral tastant [mean $(\mathrm{SD})=$ $4.7(0.7)]$. The sour and salty tastants did not differ in pleasant- 

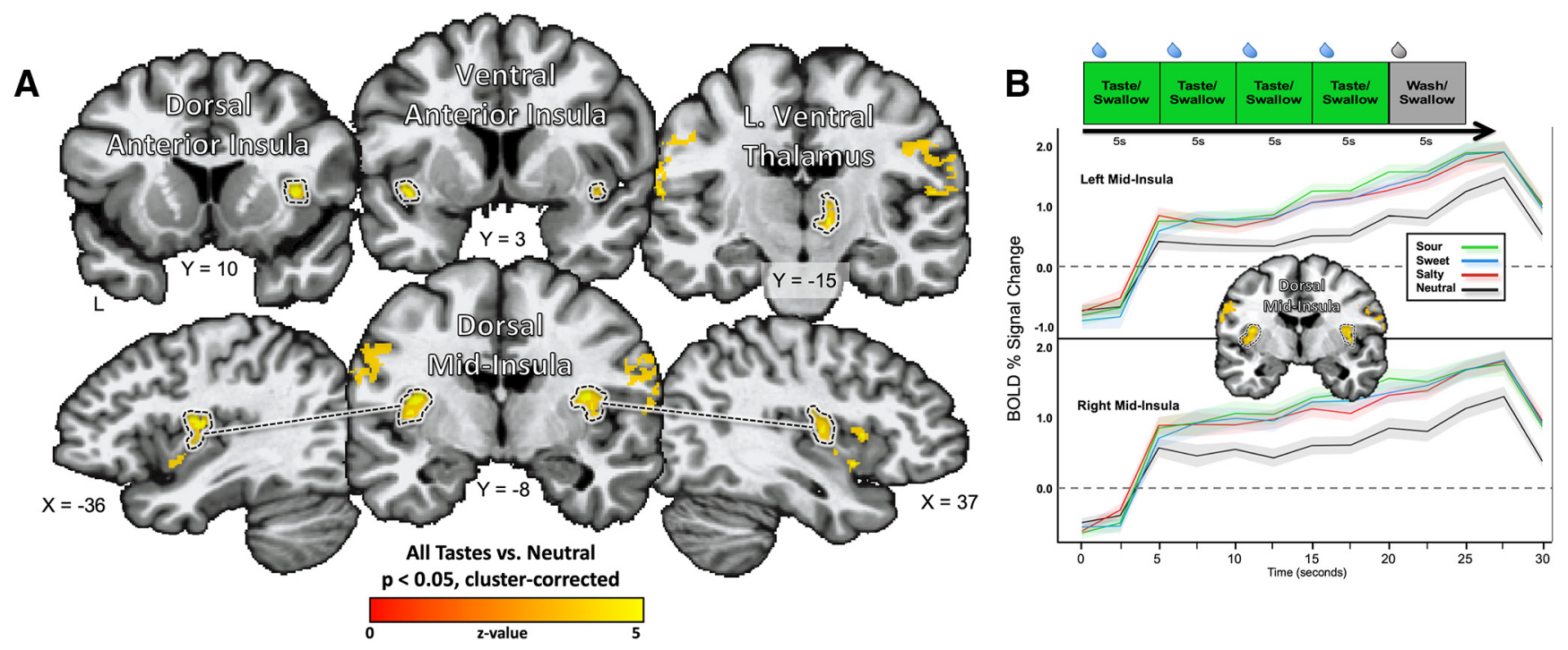

Figure 3. Taste-responsive regions of the brain. A, All tastes (sweet, sour, and salty) reliably activated taste cortex in the dorsal mid-insula, as well as primary lingual somatosensory cortex and a region of left ventral thalamus. Each of these regions has been previously identified as taste-responsive in prior human neuroimaging studies of taste (Small, 2010; Veldhuizen et al., 2011; Yeung et al., 2017). $\boldsymbol{B}$, The hemodynamic response within the bilateral mid-insula, estimated using finite impulse response functions, closely tracks the delivery of tastants during tastant blocks. The average response to each taste within the dorsal mid-insula is greater than the tasteless control, and this region exhibits no preferred activation for either taste. For results after more aggressive correction for swallowing-induced motion, see Figure 3-1, available at https://doi.org/10.1523/JNEUROSCI.1751-19.2019.f3-1.

Table 2. Brain regions responsive to tastants (vs tasteless control)

\begin{tabular}{lrrrrrr}
\hline & \multicolumn{3}{c}{ Peak coordinates TLRC } & & & Volume, \\
\cline { 2 - 4 } Anatomical location & $x$ & $y$ & $z$ & Peak Z & Cluster $p$ & $\mathrm{~mm}^{3}$ \\
\hline Right somatosensory cortex & 60 & -1 & 23 & 5.20 & $<0.01$ & 2798 \\
Left somatosensory cortex & -57 & -15 & 12 & 5.04 & $<0.01$ & 2286 \\
Left cerebellum & -15 & -60 & -22 & 5.28 & $<0.01$ & 1106 \\
Left mid-insula & -34 & -7 & 14 & 5.46 & $<0.01$ & 900 \\
Right cerebellum & 22 & -63 & -19 & 4.96 & $<0.01$ & 757 \\
Right mid-insula & 33 & -8 & 14 & 5.09 & $<0.01$ & 689 \\
Right mid-anterior insula & 35 & 11 & 7 & 5.43 & $<0.01$ & 313 \\
Right ventral thalamus & 10 & -15 & 1 & 5.08 & $<0.01$ & 257 \\
Right ventral anterior insula & 37 & 7 & -5 & 5.16 & $<0.02$ & 152 \\
\hline
\end{tabular}

For MNI coordinates, see Table 2-1, available at https://doi.org/10.1523/JNEUROSCI.1751-19.2019.t2-1.

ness [sour: mean $(\mathrm{SD})=3.7(1.5)$; salty: mean $(\mathrm{SD})=3.8(1.6)$; $p>0.99$ ]. These pleasantness and intensity ratings are comparable to those reported in previous neuroimaging studies of taste using similar concentrations (Bender et al., 2009). The average identification accuracy across all subjects was $93 \%(\mathrm{SD}=8 \%)$.

\section{Imaging results}

Univariate contrasts

All tastes compared with the tasteless control significantly activated a bilateral region of the dorsal mid-insula, located in an area in the fundus of the central insular sulcus and the overlying frontoparietal operculum (Fig. $3 a$; Table 2). In the left insula, as well as the right, activation for taste versus neutral extended ventrally from this dorsal mid-insula area down the central insular sulcus, extending toward the ventral anterior insula. A separate cluster was located in the dorsal right anterior insula, located in the fundus of the overlying frontal operculum, on the middle short insular gyrus. Outside of the insula, we observed significant activation in the bilateral postcentral gyrus, approximately in the tongue and mouth region of somatosensory cortex, as well as bilateral regions of cerebellar lobule VI, regions associated with orosensory and motor movements (Suzuki et al., 2003). Finally, we observed activation for all tastes in a cluster of the right ventral thalamus, in the approximate location of the gustatory thalamic nucleus (Fig. 3a; Table 2). These results were obtained using nonparametric cluster-size correction for multiple comparisons over the whole scan volume.

Taste specificity analyses

A series of whole-brain conjunction analyses was performed to identify voxels within the brain that were preferentially activated by each taste greater than both other tastes as well as the tasteless control. After correction for multiple comparisons using threshold-free cluster-enhancement and voxelwise conjunction analysis, we did not identify any voxels within the insula or the wider brain exhibiting a significant preference for either sweet, sour, or salty taste (Fig. $4 a$; uncorrected maps presented for display purposes only). In another analysis approach, we did not identify any regions of the brain that exhibited a main effect of taste quality (among sweet, sour, and salty), after correction for multiple comparisons.

\section{Subject-level results}

At the subject-level, a similar series of taste specificity analyses identified distributions of voxels within the insula that exhibited a selective preference for specific tastes. However, the distribution of these voxels was not consistent across subjects, nor were the dominant preferences in any consistent pattern (Fig. 4b). To identify whether these taste-selective responses reflected a consistent spatial organization at the subject-level, we brought back a subset of our subjects for a second session of the Taste Perception task $(N=5)$. We observed that, within individual subjects, the patterns of taste-selective responses identified via the conjunction analyses did not remain consistent from day to day (Fig. 4b).

\section{MVPA results}

To identify whether taste-selective activity patterns are present within insula regions broadly responsive to multiple tastes, we performed MVPA using the mid-insula ROIs identified (Fig. 3a). We were able to reliably classify between sweet, salty, and sour tastants within the bilateral dorsal mid-insula with an average accuracy of $\sim 62 \%$ (i.e., $12 \%$ above chance; all pairwise $p$ values, $p<0.001$; Fig. $5 a$ ). In a supplemental analysis, we also performed 


\section{A Group level taste conjunctions}

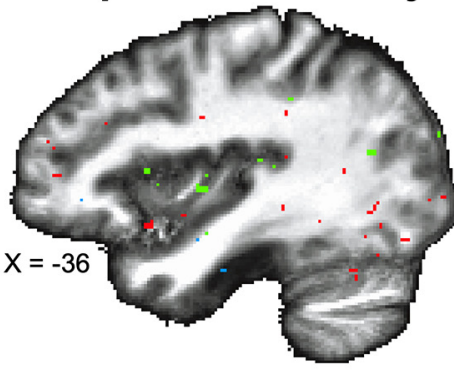

Taste specificity contrasts, $p<0.05-$ uncorrected
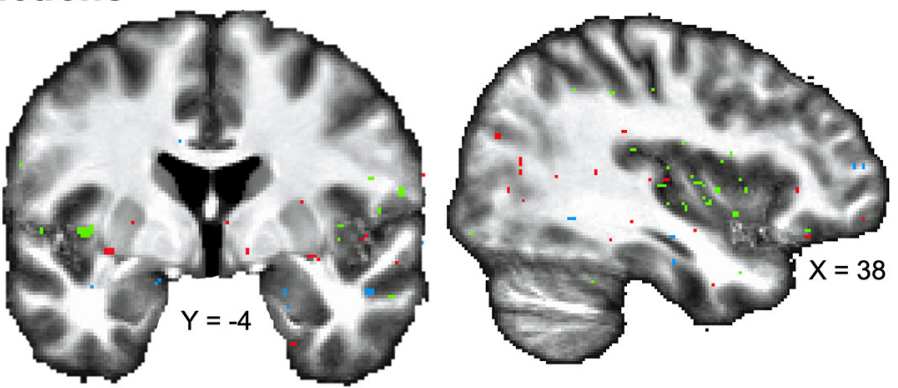

Conjunction: Sweet $>$ Sour \& Sweet $>$ Salty \& Sweet $>$ Neutral Conjunction: Sour $>$ Sweet \& Sour $>$ Salty \& Sour $>$ Neutral Conjunction: Salty $>$ Sour \& Salty $>$ Sweet \& Salty $>$ Neutral

B Subject-level taste conjunctions

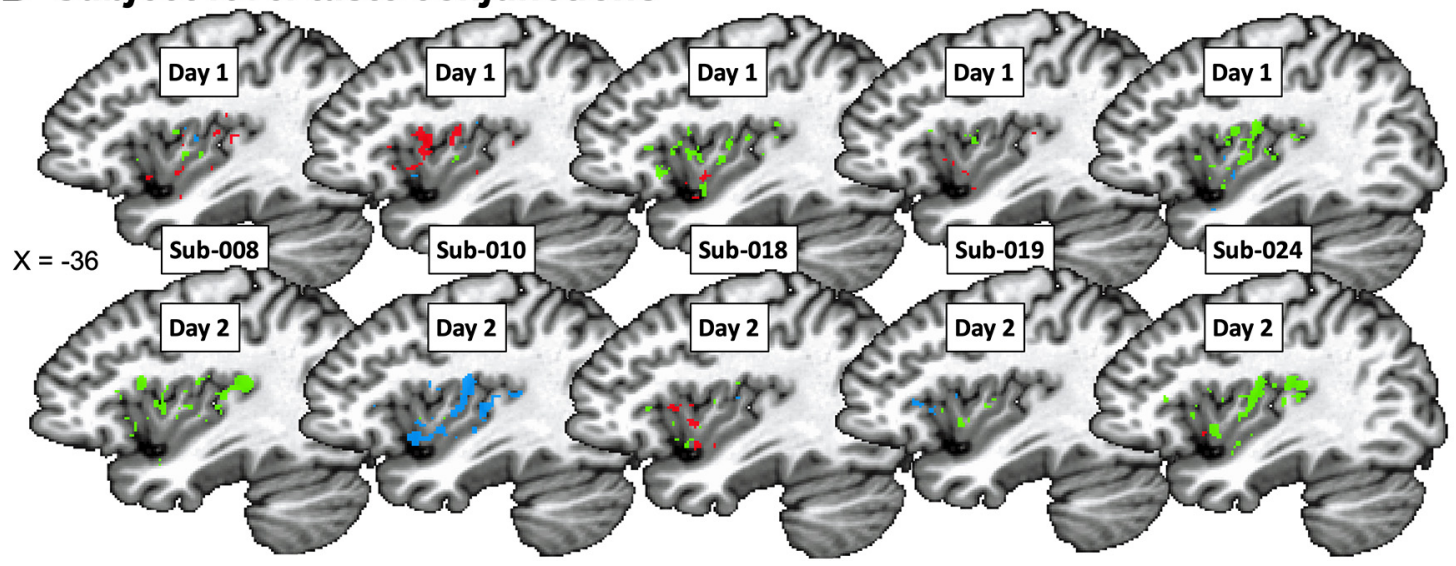

Figure 4. Conjunction analyses of taste contrasts provide no clear evidence of topographical organization. $A$, At the group level, whole-brain conjunctions of taste contrasts, using corrected statistical contrasts, did not identify any voxels within the insula or the wider brain exhibiting a significant preference for either sweet, sour, or salty taste. $\boldsymbol{B}$, Specificity maps from five subjects who were scanned on two separate sessions. Not only did specificity maps vary greatly between subjects, the patterns of taste-selective responses identified did not remain consistent within individual subjects from day to day. Images above show uncorrected specificity maps at low statistical threshold $(p<0.05)$.

the ROI analyses using leave-two-runs-out cross-validation. The results of this procedure did not substantively differ from the results using leave-one-out-cross-validation.

The multivariate searchlight analysis identified a number of brain regions that exhibited significant, above chance classification accuracy for discriminating between tastes. Those regions included primary sensory areas such as bilateral regions of the dorsal mid-insular cortex, somatosensory cortex, and piriform cortex. Additionally, we also were able to reliably classify between distinct tastes within brain regions involved in affect and reward, including the bilateral amygdala, the left orbitofrontal cortex (BA $11 \mathrm{~m})$, the mediodorsal thalamus, the dorsal striatum, and the subgenual prefrontal cortex (BA25; Fig. 6; for a list of these regions and descriptive and statistical data, see Table 3 ).

\section{Pleasantness analyses}

We also attempted to identify the effect of self-reported pleasantness ratings on the hemodynamic response to tastants. We performed both a group-level $t$ test of parametrically-modulated hemodynamic response functions as well as a group-level linear mixed-effects regression model. After correction for multiple comparisons, neither approach was able to identify any brain regions exhibiting a reliable relationship between pleasantness ratings and tastant response. Additionally, removal of the effect of pleasantness ratings, using the group-level LME model, did not noticeably diminish the size of clusters present with our main univariate contrast (i.e., taste vs tasteless; Fig. 3).

\section{Discussion}

Prior evidence from optical imaging studies in rodent models has suggested that tastes are represented in topographically distinct areas within the insular cortex (Chen et al., 2011). However, studies in nonhuman primates instead have suggested that taste within the insula is represented within dispersed populations of cortical neurons, without any clear spatial organization (Scott and Plata-Salamán, 1999). Human neuroimaging studies of taste to date have largely been conducted using low-resolution functional neuroimaging methods, which may not have the power to discriminate between distinct taste-specific regions within insular taste cortex. To discriminate between the competing topographical and population coding models, we examined the spatial representation of multiple tastes within the human brain using ultra-high resolution fMRI at high magnetic field strength. In agreement with previous human neuroimaging studies of taste (Small, 2010; Veldhuizen et al., 2011; Yeung et al., 2017), sweet, sour, and salty tastant solutions (vs a tasteless solution) activated regions of the anterior and mid-insular cortex. However, we did not observe any evidence that any region within the insula, or in the rest of the brain, exhibited a clear preference for a specific taste. 


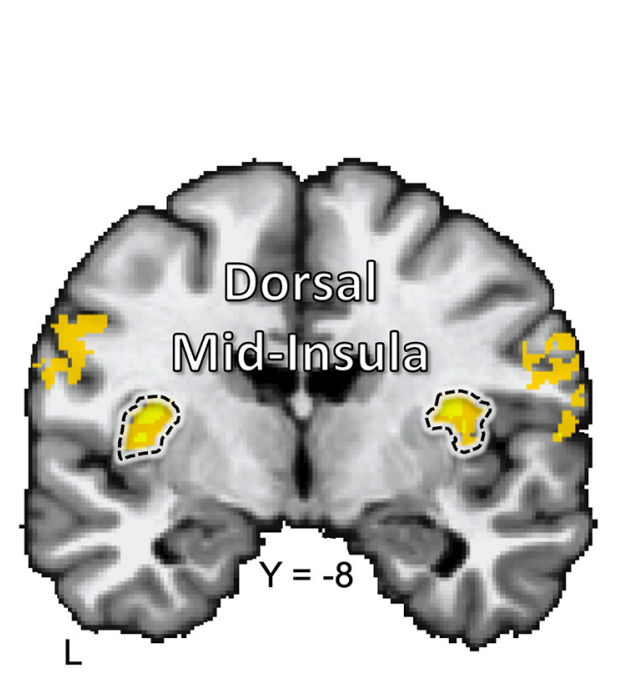

B

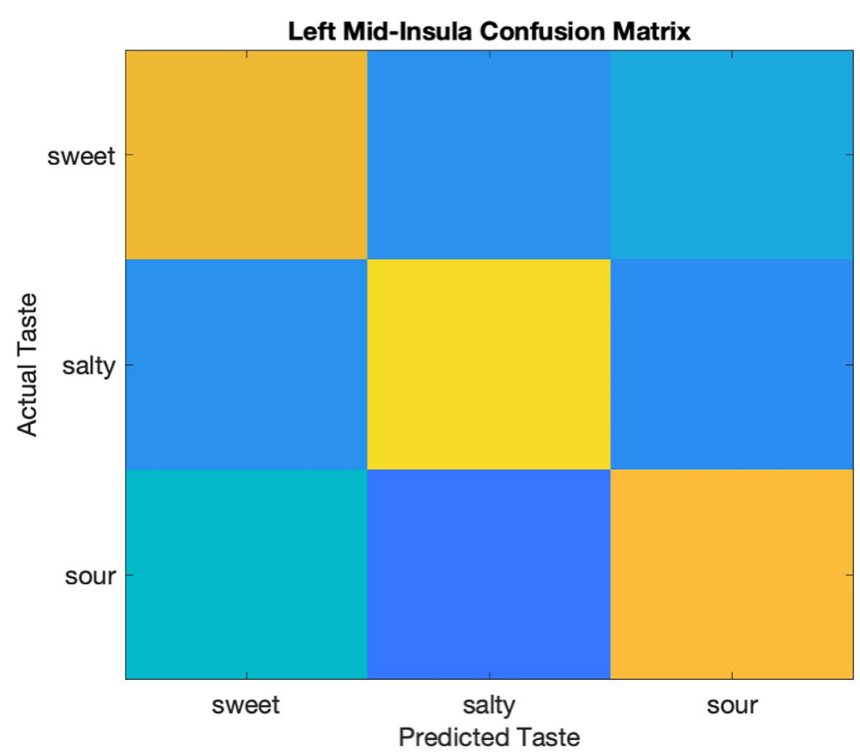

\section{A}

Pairwise Classification Accuracy: Dorsal Mid-Insula ROIs

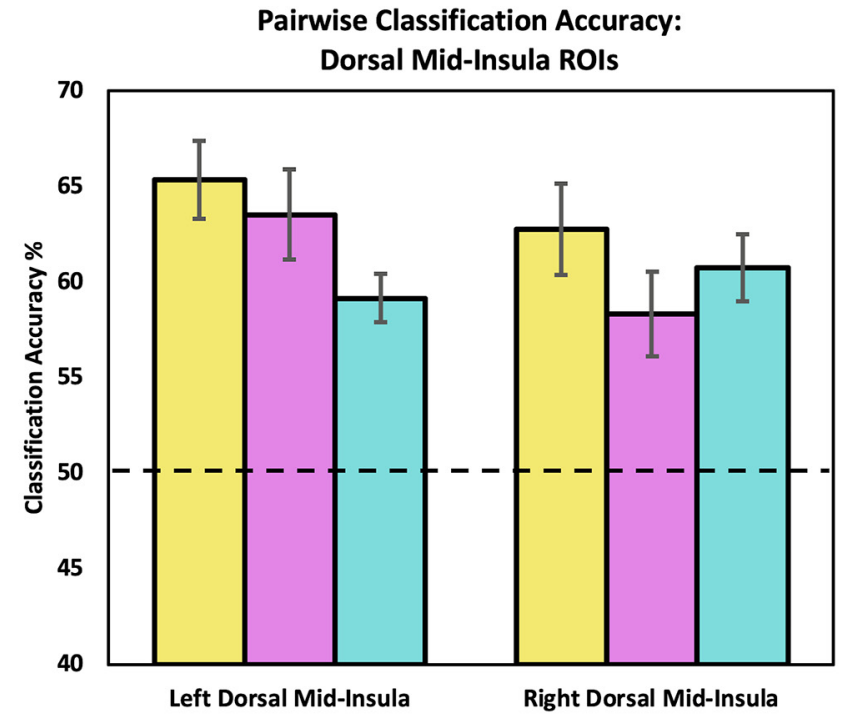

口Salty vs. Sour $\square$ Salty vs. Sweet $\square$ Sweet vs. Sour

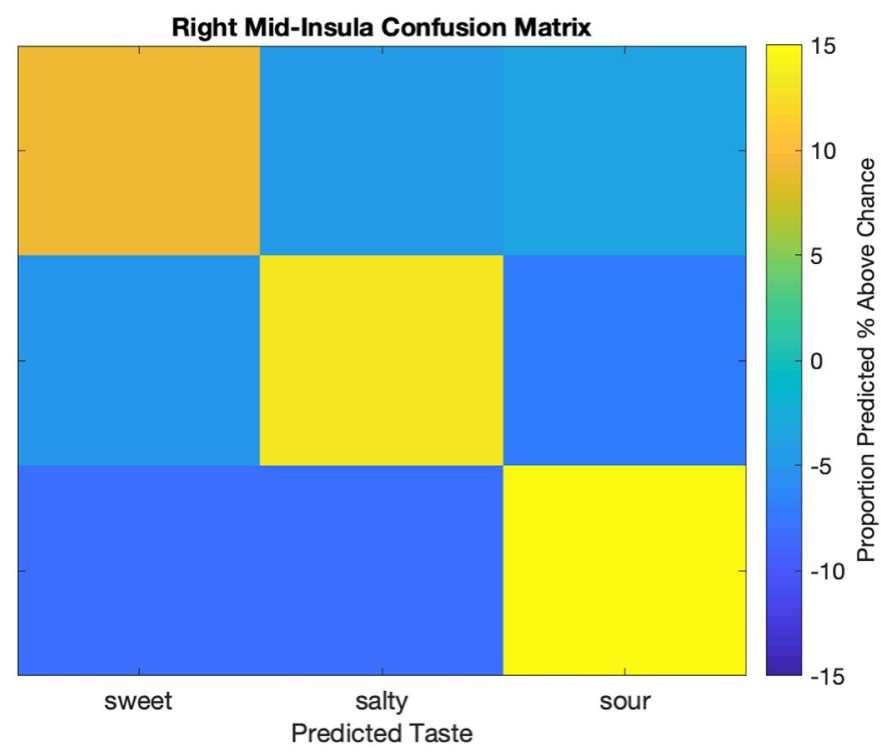

Figure 5. Multivariate pattern analyses decode taste quality within primary taste cortex. Multivariate pattern analyses run within the bilateral mid-insula, a region broadly responsive to multiple tastes (Fig. 3) show high accuracy for classifying between distinct tastes, as seen in (A) pairwise classification accuracy plots and (B) multiclass confusion matrices. R0I, region of interest.

One previous fMRI study that examined taste representation in the insula reported that patterns of taste-specific responses were highly variable across subjects, but suggested that those patterns were stable over time within subjects (Schoenfeld et al., 2004). To examine this possibility of a stable individual-level topography, we also examined the tastant responses of a subset of subjects which were scanned during two separate sessions. Using the same type of conjunction analyses we used at the group level, we observed that the patterns of taste-specific responses were not only highly variable across subjects, but within subjects as well. Although we were unable to rule out the possibility of a microscale organization of taste specific neurons within gustatory cortex, such as that observed in previous studies of rodents (Chen et al., 2011), we find little evidence for discrete topographical regions for specific tastes in humans at the spatial scales observable with ultra-high resolution fMRI.

However, using multivariate pattern analysis at those same spatial scales, we were nonetheless able to discriminate between the responses to distinct tastes within the insula. Using an MVPA searchlight approach (Kriegeskorte et al., 2006), we identified that the bilateral dorsal mid-insula reliably classified between distinct tastes with an accuracy significantly greater than chance. These results support a model of insular functional organization wherein taste quality is encoded by distributed spatial patterns within primary gustatory regions of the insular cortex, like that observed for olfaction in piriform cortex (Howard et al., 2009; Stettler and Axel, 2009). These activity patterns may be represented either in dispersed ensembles of cortical neurons specifically tuned to individual tastes (i.e., labeled-lines), by a combinatorial code within cortical neurons broadly tuned to multiple tastes (Simon et al., 2006), or by some mixture of broadly-tuned and specialist neurons (Roper and Chaudhari, 2017).

The actual location of the human primary gustatory area in the insula has also been the subject of some controversy. Many researchers have argued that the far anterior region of the dorsal insula is the likeliest candidate, given the location of area $\mathrm{G}$ in the nonhuman primate insula (Rolls, 2016). However, numerous human neuroimaging studies over the past 15 years have impli- 


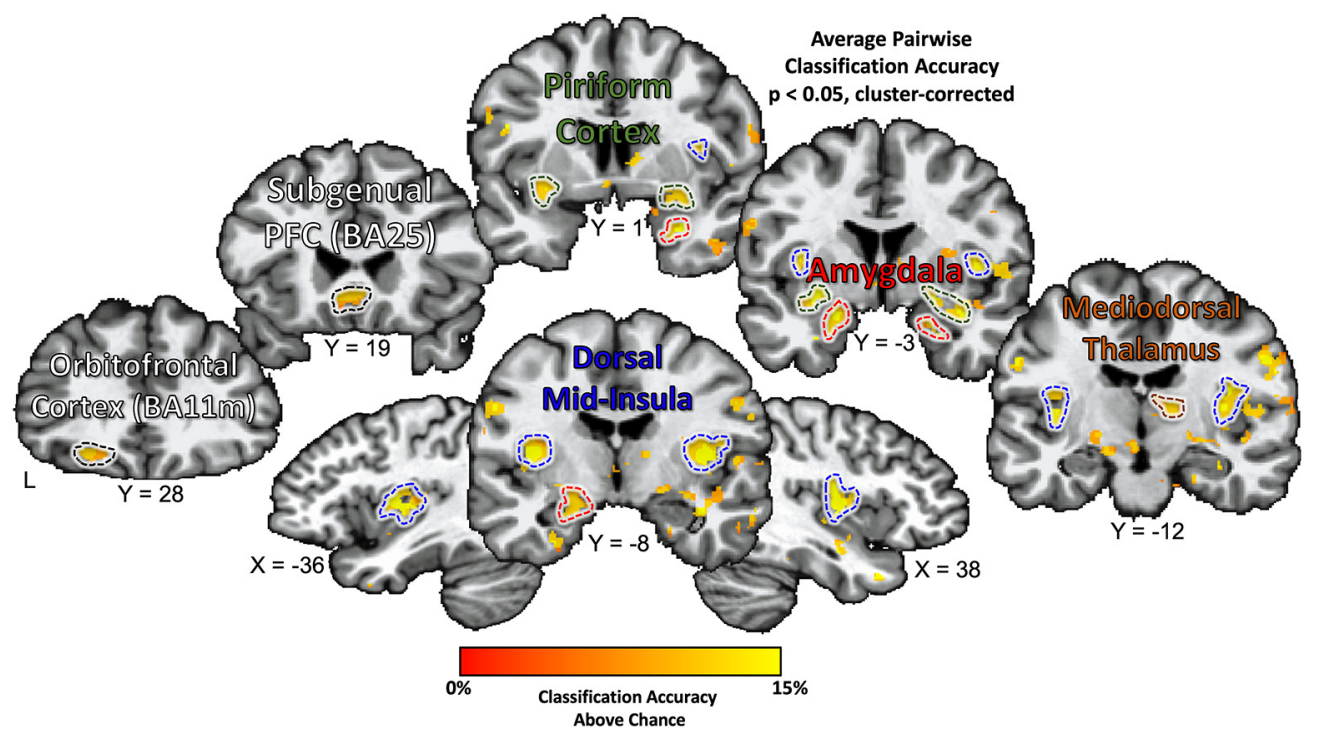

Figure 6. Multivariate pattern analyses decode taste quality within brain regions involved in taste perception and reward. Several regions of the brain were identified using a multivariate searchlight analysis, trained to distinguish between sweet, salty, and sour tastes. Among those regions were primary sensory regions for taste, the bilateral dorsal mid-insula, and smell, the bilateral piriform cortex, as well as limbic regions involved in affect and reward, such as the amygdala, orbitofrontal cortex, subgenual prefrontal cortex, mediodorsal thalamus. For results after more aggressive correction for swallowing-induced motion, see Figure 6-1, available at https://doi.org/10.1523/JNEUROSCl.1751-19.2019.f6-1. BA, Brodmann area.

Table 3. Brain regions where multivoxel patterns reliably discriminate between sweet, salty, and sour tastants

\begin{tabular}{|c|c|c|c|c|c|c|c|}
\hline \multirow{2}{*}{$\begin{array}{l}\text { Location (multiple anatomical } \\
\text { regions within a cluster are } \\
\text { separated by semicolons) }\end{array}$} & \multicolumn{3}{|c|}{ Peak coordinates } & \multirow[b]{2}{*}{ Peak Z } & \multirow{2}{*}{$\begin{array}{l}\text { Peak } \\
\text { ACC, } \%\end{array}$} & \multirow[b]{2}{*}{ Cluster $p$} & \multirow{2}{*}{$\begin{array}{l}\text { Volume, } \\
\mathrm{mm}^{3}\end{array}$} \\
\hline & $x$ & $y$ & $Z$ & & & & \\
\hline $\begin{array}{l}\text { Right mid-insula; right } \\
\text { parietal operculum }\end{array}$ & 36 & -9 & 10 & 5.65 & 14.00 & $<0.01$ & 2964 \\
\hline Right piriform cortex & 23 & -3 & -7 & 5.26 & 13.10 & $<0.01$ & 1510 \\
\hline Left amygdala & -20 & -4 & -13 & 5.47 & 13.90 & $<0.01$ & 1337 \\
\hline Right pons & 7 & -16 & -24 & 5.18 & 12.70 & $<0.01$ & 1109 \\
\hline Left somatosensory cortex & -52 & -15 & 29 & 5.39 & 11.50 & $<0.01$ & 1085 \\
\hline Left mid-insula & -34 & -4 & 9 & 5.13 & 14.08 & $<0.01$ & 931 \\
\hline Right hippocampus & 19 & -23 & -12 & 4.86 & 11.50 & $<0.01$ & 757 \\
\hline Right somatosensory cortex & 53 & -11 & 30 & 5.30 & 12.85 & $<0.01$ & 755 \\
\hline Right parahippocampal gyrus & 28 & -27 & -19 & 4.80 & 11.80 & $<0.01$ & 679 \\
\hline Left periaqueductal gray & -5 & -27 & -11 & 4.78 & 10.60 & $<0.01$ & 662 \\
\hline Subgenual prefrontal cortex & 8 & 25 & -3 & 5.05 & 13.40 & $<0.01$ & 655 \\
\hline Left cerebellum & -20 & -26 & -27 & 5.06 & 10.30 & $<0.01$ & 534 \\
\hline Right amygdala & 28 & 2 & -22 & 4.40 & 13.20 & $<0.01$ & 491 \\
\hline Right dorsal lateral thalamus & 14 & -10 & 9 & 5.26 & 11.20 & $<0.01$ & 429 \\
\hline Left temporal pole & -27 & 11 & -29 & 5.35 & 13.80 & $<0.01$ & 396 \\
\hline Left lateral orbitofrontal cortex & -20 & 28 & -10 & 5.11 & 10.90 & $<0.01$ & 359 \\
\hline Left dorsal striatum & -16 & 7 & 17 & 4.78 & 10.90 & $<0.01$ & 223 \\
\hline Left ventral tegmental area & -4 & -10 & -9 & 4.68 & 11.03 & $<0.02$ & 219 \\
\hline Right temporal pole & 40 & 13 & -27 & 4.61 & 12.08 & $<0.02$ & 207 \\
\hline Right lingual gyrus & 9 & -63 & 0 & 4.48 & 11.90 & $<0.02$ & 200 \\
\hline Right mediodorsal thalamus & 4 & -17 & 6 & 5.29 & 9.93 & $<0.02$ & 195 \\
\hline Right hippocampus & 25 & -29 & -4 & 4.58 & 9.48 & $<0.02$ & 188 \\
\hline Right mid-cingulate cortex & 7 & 9 & 27 & 4.99 & 8.56 & $<0.03$ & 175 \\
\hline Right inferior temporal gyrus & 58 & -7 & -21 & 4.90 & 9.93 & $<0.03$ & 173 \\
\hline Right caudate nucleus & 9 & 2 & 6 & 4.40 & 11.60 & $<0.03$ & 173 \\
\hline
\end{tabular}

For MNI coordinates, see Table 3-1, available at https://doi.org/10.1523/JNEUROSCI.1751-19.2019.t3-1.

cated the dorsal anterior insula as playing a more domain-general role in cognition and attention (Kurth et al., 2010; Nelson et al., 2010), and many consider the human anterior insula to be a newly developed cortical structure (Craig, 2009), perhaps because of the expansion of language faculties in our species (Nieuwenhuys, 2012). The effects of task context, such as whether subjects identify or evaluate a taste during scanning, have also been shown to affect the spatial representation of that taste within the insula (Bender et al., 2009). Nevertheless, a large number of human neuroimaging studies of taste, incorporating multiple imaging modalities (Kobayakawa et al., 1999, 2005; Veldhuizen et al., 2007; Iannilli et al., 2014), as well as cortical electrode stimulation studies in presurgical patients (Mazzola et al., 2017), point to the mid-to-posterior dorsal insula as the location of human primary gustatory cortex. In the present study, we identified regions of the dorsal mid-insula, anterior ventral insula, and midanterior insula that exhibited a significant activation to taste versus tasteless solutions, consistent with meta-analyses of human neuroimaging studies (Veldhuizen et al., 2011; Yeung et al., 2017). Of those regions, the bilateral dorsal mid-insula regions exhibited the most consistent ability to discriminate between different tastant solutions, suggesting a focal role for the dorsal midinsula in the primary sensory processing of taste.

One recent human neuroimaging study, which was acquired using lower resolution fMRI $\left(36.75 \mathrm{~mm}^{3}\right.$ at $3 \mathrm{~T} ; 8 \mathrm{~mm}^{3}$ at $7 \mathrm{~T}$ vs $1.73 \mathrm{~mm}^{3}$ for the present study) and within the context of a hedonic evaluation task, also provided evidence that multivariate methods could be used to classify tastes within the insula (Chikazoe et al., 2019). In agreement with the results of Chikazoe et al. (2019), we identified regions of the mid-insula that reliably classify between different tastes. In contrast to their results, however, we identified other regions of the brain that exhibited spatial activity patterns that discriminated between different tastes. These regions included other cortical sensory regions such as the piriform cortex, involved in olfactory processing (Sobel et al., 2000) as well as regions involved in various aspects of food sensation and reward, such as the bilateral amygdala, the orbitofrontal cortex, the dorsal striatum, and the mediodorsal thalamus. These results suggest that the presence of multivariate patterns supporting information related to taste is not a unique feature of insular taste cortex, as previously suggested (Chikazoe et al., 2019). Notably, the amygdala, orbitofrontal cortex and striatum are all downstream regions in the taste pathway (Scott and PlataSalamán, 1999; Rolls, 2005), that receive primary (or secondary) projections from gustatory cortex, and that play different roles in food reward, aversion, and value based decision-making (Krin- 
gelbach et al., 2003; Kringelbach, 2005; Rolls, 2005; Saez et al., 2017). The involvement of the amygdala in the perception of basic tastes has been demonstrated in patients undergoing amygdala resection, who exhibit increased sensitivity to sour taste and greater perceived taste intensity (Small et al., 1997). The activity of the amygdala, especially the central nucleus and basolateral amygdala, is strongly associated with conditioned taste aversion (Reilly and Bornovalova, 2005). The OFC, in concert with the amygdala and mediodorsal thalamus, are thought to represent the moment-to-moment value of environment stimuli and sensory experiences, informed by the body's current state (Rudebeck and Murray, 2014). Importantly, although these MVPA results may indicate that these regions contain reliable information about taste quality, they do not indicate the precise nature of that information (Hebart and Baker, 2018). As these regions play key roles in taste perception and normative responses to food, the multivariate taste-specific patterns identified within them might reflect codes for taste quality, or they might reflect information about other stimulus dimensions such as the pleasantness or aversiveness of these distinct tastes.

\section{Limitations}

Within a supplemental analysis, we attempted to account for any effect of swallowing-induced head motion during the task by including a regressor for the onset of each swallowing event within the subject-level regression model. We also included another parametrically-modulated regressor within that model to account for the magnitude of head motion estimated for each swallowing event. We reanalyzed our imaging data using these regressors at the subject, group, and decoding level, and our results remained consistent and largely unchanged (see Extended Data). However, although these measures may help to account for any head motion-related differences in swallowing, they cannot completely account for any neural differences due to differing oromotor actions during swallowing.

Pleasantness is one of the defining features of the sense of taste, with some tastes, such as sweet, being inherently more pleasant than others, such as bitter. Within the present study, we attempted to minimize the effect of taste pleasantness, in an effort to dissociate taste pleasantness from taste quality. To this end, we specifically used mild concentrations of sweet, sour, and salty tastants and did not use a bitter tastant. We examined the effect of pleasantness upon tastant responses using two different approaches, one in which participants' pleasantness ratings were used to account for trial-by-trial variance (i.e., amplitude modulation regression) and one in which ratings were used to account for any remaining variance at the group level. After correction for multiple comparisons, neither approach provided evidence for an effect of participants' self-reported pleasantness ratings upon the hemodynamic response to tastes. Although a complete dissociation of taste quality and taste pleasantness may not be experimentally feasible, within the context of this study, in which tastes were only mildly pleasant or mildly aversive, variability in pleasantness did not account for a significant amount of variability in taste response.

\section{Conclusion}

We set out to distinguish between two competing theories of taste representation in the insula using ultra-high resolution $\mathrm{AMRI}$ at high magnetic field strength. We identified a strong overlap in the activity for all three tastes in previously identified gustatory regions of the insula. We did not identify any regions within the insula or the wider brain that exhibited a preference for specific tastes. However, we were able to decode taste identity in a consistent manner within primary gustatory insula and other brain regions associated with food perception and reward. This suggests that taste quality exists in a distributed pattern across multiple voxels within gustatory cortex, potentially resulting from a population code among dispersed populations of tasteresponsive cortical neurons. This information is then presumably passed down through a network of cortical and subcortical regions involved in appetitive and behavioral responses to food.

\section{Reference}

Avery JA, Kerr KL, Ingeholm JE, Burrows K, Bodurka J, Simmons WK (2015) A common gustatory and interoceptive representation in the human mid-insula. Hum Brain Mapp 36:2996-3006.

Avery JA, Gotts SJ, Kerr KL, Burrows K, Ingeholm JE, Bodurka J, Martin A, Kyle Simmons W (2017) Convergent gustatory and viscerosensory processing in the human dorsal mid-insula. Hum Brain Mapp 38:2150-2164.

Avery JA, Ingeholm JE, Wohltjen S, Collins M, Riddell CD, Gotts SJ, Kenworthy L, Wallace GL, Simmons WK, Martin A (2018) Neural correlates of taste reactivity in autism spectrum disorder. Neuroimage 19:38-46.

Beckstead RM, Morse JR, Norgren R (1980) The nucleus of the solitary tract in the monkey: projections to the thalamus and brain stem nuclei. J Comp Neurol 190:259-282.

Bender G, Veldhuizen MG, Meltzer JA, Gitelman DR, Small DM (2009) Neural correlates of evaluative compared with passive tasting. Eur J Neurosci 30:327-338.

Chandrashekar J, Hoon MA, Ryba NJ, Zuker CS (2006) The receptors and cells for mammalian taste. Nature 444:288-294.

Chen G, Saad ZS, Britton JC, Pine DS, Cox RW (2013) Linear mixed-effects modeling approach to FMRI group analysis. Neuroimage 73:176-190.

Chen X, Gabitto M, Peng Y, Ryba NJ, Zuker CS (2011) A gustotopic map of taste qualities in the mammalian brain. Science 333:1262-1266.

Chikazoe J, Lee DH, Kriegeskorte N, Anderson AK (2019) Distinct representations of basic taste qualities in human gustatory cortex. Nat Commun 10:1048.

Craig AD (2009) How do you feel-now? The anterior insula and human awareness. Nat Rev Neurosci 10:59-70.

Eklund A, Nichols TE, Knutsson H (2016) Cluster failure: why fMRI inferences for spatial extent have inflated false-positive rates. Proc Natl Acad Sci U S A 113:7900-7905.

Hebart MN, Baker CI (2018) Deconstructing multivariate decoding for the study of brain function. Neuroimage 180:4-18.

Hebart MN, Görgen K, Haynes JD (2014) The decoding toolbox (TDT): a versatile software package for multivariate analyses of functional imaging data. Front Neuroinform 8:88.

Howard JD, Plailly J, Grueschow M, Haynes JD, Gottfried JA (2009) Odor quality coding and categorization in human posterior piriform cortex. Nat Neurosci 12:932-938.

Iannilli E, Noennig N, Hummel T, Schoenfeld AM (2014) Spatio-temporal correlates of taste processing in the human primary gustatory cortex. Neuroscience 273:92-99.

Kaskan PM, Dean AM, Nicholas MA, Mitz AR, Murray EA (2019) Gustatory responses in macaque monkeys revealed with fMRI: comments on taste, taste preference, and internal state. Neuroimage 184:932-942.

Kobayakawa T, Ogawa H, Kaneda H, Ayabe-Kanamura S, Endo H, Saito S (1999) Spatio-temporal analysis of cortical activity evoked by gustatory stimulation in humans. Chem Senses 24:201-209.

Kobayakawa T, Wakita M, Saito S, Gotow N, Sakai N, Ogawa H (2005) Location of the primary gustatory area in humans and its properties, studied by magnetoencephalography. Chem Senses 30:i226-i227.

Kriegeskorte N, Goebel R, Bandettini P (2006) Information-based functional brain mapping. Proc Natl Acad Sci U S A 103:3863-3868.

Kringelbach ML (2005) The human orbitofrontal cortex: linking reward to hedonic experience. Nat Rev Neurosci 6:691-702.

Kringelbach ML, O'Doherty J, Rolls ET, Andrews C (2003) Activation of the human orbitofrontal cortex to a liquid food stimulus is correlated with its subjective pleasantness. Cereb Cortex 13:1064-1071.

Kurth F, Zilles K, Fox PT, Laird AR, Eickhoff SB (2010) A link between the systems: functional differentiation and integration within the human insula revealed by meta-analysis. Brain Struct Funct 214:519-534. 
Mazzola L, Mauguière F, Isnard J (2017) Electrical stimulations of the human insula. J Clin Neurophysiol 34:307-314.

Nelson SM, Dosenbach NU, Cohen AL, Wheeler ME, Schlaggar BL, Petersen SE (2010) Role of the anterior insula in task-level control and focal attention. Brain Struct Funct 214:669-680.

Nieuwenhuys R (2012) The insular cortex: a review. Prog Brain Res 195: 123-163.

Prinster A, Cantone E, Verlezza V, Magliulo M, Sarnelli G, Iengo M, Cuomo R, Di Salle F, Esposito F (2017) Cortical representation of different taste modalities on the gustatory cortex: a pilot study. PLoS One 12:e0190164.

Pritchard TC, Hamilton RB, Morse JR, Norgren R (1986) Projections of thalamic gustatory and lingual areas in the monkey, Macaca fascicularis. J Comp Neurol 244:213-228.

Reilly S, Bornovalova MA (2005) Conditioned taste aversion and amygdala lesions in the rat: a critical review. Neurosci Biobehav Rev 29:1067-1088.

Rolls ET (2005) Taste, olfactory, and food texture processing in the brain, and the control of food intake. Physiol Behav 85:45-56.

Rolls ET (2016) Functions of the anterior insula in taste, autonomic, and related functions. Brain Cogn 110:4-19.

Roper SD, Chaudhari N (2017) Taste buds: cells, signals and synapses. Nat Rev Neurosci 18:485-497.

Rudebeck PH, Murray EA (2014) The orbitofrontal oracle: cortical mechanisms for the prediction and evaluation of specific behavioral outcomes. Neuron 84:1143-1156.

Saez RA, Saez A, Paton JJ, Lau B, Salzman CD (2017) Distinct roles for the amygdala and orbitofrontal cortex in representing the relative amount of expected reward. Neuron 95:70-77.e3.

Schoenfeld MA, Neuer G, Tempelmann C, Schüßler K, Noesselt T, Hopf JM, Heinze HJ (2004) Functional magnetic resonance tomography correlates of taste perception in the human primary taste cortex. Neuroscience 127:347-353.

Scott TR, Giza BK (2000) Issues of gustatory neural coding: where they stand today. Physiol Behav 69:65-76.

Scott TR, Plata-Salamán CR (1999) Taste in the monkey cortex. Physiol Behav 67:489-511.

Scott TR, Plata-Salamán CR, Smith VL, Giza BK (1991) Gustatory neural coding in the monkey cortex: stimulus intensity. J Neurophysiol 65: $76-86$.

Simmons WK, Avery JA, Barcalow JC, Bodurka J, Drevets WC, Bellgowan P (2013a) Keeping the body in mind: insula functional organization and functional connectivity integrate interoceptive, exteroceptive, and emotional awareness. Hum Brain Mapp 34:2944-2958.

Simmons WK, Rapuano KM, Kallman SJ, Ingeholm JE, Miller B, Gotts SJ, Avery JA, Hall KD, Martin A (2013b) Category-specific integration of homeostatic signals in caudal but not rostral human insula. Nat Neurosci 16:1551-1552.

Simon SA, de Araujo IE, Gutierrez R, Nicolelis MA (2006) The neural mechanisms of gustation: a distributed processing code. Nat Rev Neurosci 7:890-901.

Small DM (2010) Taste representation in the human insula. Brain Struct Funct 214:551-561.

Small DM, Jones-Gotman M, Zatorre RJ, Petrides M, Evans AC (1997) A role for the right anterior temporal lobe in taste quality recognition. J Neurosci 17:5136-5142.

Smith SM, Nichols TE (2009) Threshold-free cluster enhancement: addressing problems of smoothing, threshold dependence and localisation in cluster inference. Neuroimage 44:83-98.

Sobel N, Prabhakaran V, Zhao Z, Desmond JE, Glover GH, Sullivan EV, Gabrieli JD (2000) Time course of odorant-induced activation in the human primary olfactory cortex. J Neurophysiol 83:537-551.

Stettler DD, Axel R (2009) Representations of odor in the piriform cortex. Neuron 63:854-864.

Suzuki M, Asada Y, Ito J, Hayashi K, Inoue H, Kitano H (2003) Activation of cerebellum and basal ganglia on volitional swallowing detected by functional magnetic resonance imaging. Dysphagia 18:71-77.

Tomchik SM, Berg S, Kim JW, Chaudhari N, Roper SD (2007) Breadth of tuning and taste coding in mammalian taste buds. J Neurosci 27: 10840-10848

Veldhuizen MG, Bender G, Constable RT, Small DM (2007) Trying to detect taste in a tasteless solution: modulation of early gustatory cortex by attention to taste. Chem Senses 32:569-581.

Veldhuizen MG, Albrecht J, Zelano C, Boesveldt S, Breslin P, Lundström JN (2011) Identification of human gustatory cortex by activation likelihood estimation. Hum Brain Mapp 32:2256-2266.

Yeung AWK, Goto TK, Leung WK (2017) Basic taste processing recruits bilateral anteroventral and middle dorsal insulae: an activation likelihood estimation meta-analysis of fMRI studies. Brain Behav 7:e00655-712. 